\title{
Enantioselective Electrochemical Lactonization Using Chiral lodoarenes as Mediators
}

\author{
Wen-Chao Gao' \\ Zi-Yue Xiong \\ Shafigh Pirhaghani \\ Thomas Wirth* (D) \\ School of Chemistry, Cardiff University, Park Place, Cardiff, \\ CF10 3AT, UK \\ wirth@cf.ac.uk
}

Published as part of the 50 Years SYNTHESIS - Golden Anniversary Issue

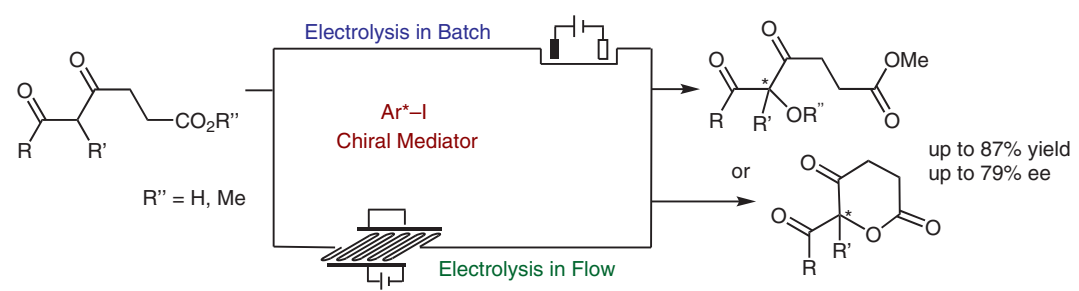

\author{
Received: 07.09.2018 \\ Accepted after revision: 04.10.2018 \\ Published online: 25.10 .2018 \\ DOI: 10.1055/s-0037-1610373; Art ID: ss-2018-z0601-op \\ License terms: cc)
}

Abstract The enantioselective electrochemical lactonization of diketo acid derivatives using chiral iodoarenes as redox mediators is reported for the first time. Good to high stereoselectivities are observed in the lactonization and also in intermolecular $\alpha$-alkoxylations of diketo ester derivatives. This enantioselective process was then adapted to an electrochemical flow microreactor where only small amounts of supporting electrolyte were necessary.

Key words electrolysis, flow microreactors, hypervalent iodine, lactonization, stereoselective synthesis

The utility of chiral hypervalent iodine compounds for enantioselective oxidative reactions represents an important and interesting area in organic chemistry. ${ }^{2}$ Specifically, as a versatile and general oxidation system, the combination of $m C P B A$ as the stoichiometric oxidant and chiral iodoarenes as catalysts has been successfully applied to various transformations, including the asymmetric difunctionalization of alkenes, ${ }^{3}$ the stereoselective dearomatization of phenol or naphthol derivatives, ${ }^{4}$ enantioselective oxidative rearrangement ${ }^{5}$ and the enantioselective synthesis of spirooxindole derivatives. ${ }^{6}$ Nevertheless, although enantioselective oxidative lactonization has been well developed via the dearomatization of phenol or naphthol derivatives using chiral iodoarenes in combination with mCPBA, direct lactone synthesis through oxidative cyclization of keto acids is underdeveloped, and only moderate enantioselectivities $(<51 \% \text { ee) were achieved (Scheme } 1 \text {, eq. } 1)^{7}$

As an efficient and environmentally friendly protocol for organic synthesis, electrochemical conversions have recently gained more attention as often an excess amount of conventional chemical oxidants and reducing reagents can be avoided. ${ }^{8}$ Although the electrochemical oxidative $\alpha$-lac- tonization of $\gamma$-keto acids has been reported using $n$-Bu $\mathrm{Bu}_{4} \mathrm{NI}$ as the mediator (Scheme 1, eq. 2), ${ }^{9}$ the enantioselective electrosynthesis of lactones is still unexplored. ${ }^{10}$ In organic electrochemistry, iodoarenes represent a versatile class of redox mediators, which can generate hypervalent iodine(III) reagents via anodic oxidation to accomplish various transformations without use of a stoichiometric oxidant such as $m C P B A$. The first electrolysis of iodoarenes described the synthesis of (difluoroiodo)benzene, by Schmidt and Meinert in 1960, ${ }^{11}$ and subsequently Fuchigami has contributed to the development of different fluorination reactions in electrochemistry. ${ }^{12}$ Recently, the anodic oxidation of iodoarenes in trifluoroethanol and hexafluoroisopropanol (HFIP) has been reported by Nishiyama and Francke and their co-workers, who accomplished $\mathrm{C}-\mathrm{N}$ and $\mathrm{C}-\mathrm{O}$ bond formations. ${ }^{13}$ Some reviews in this area have recently been published. ${ }^{14}$

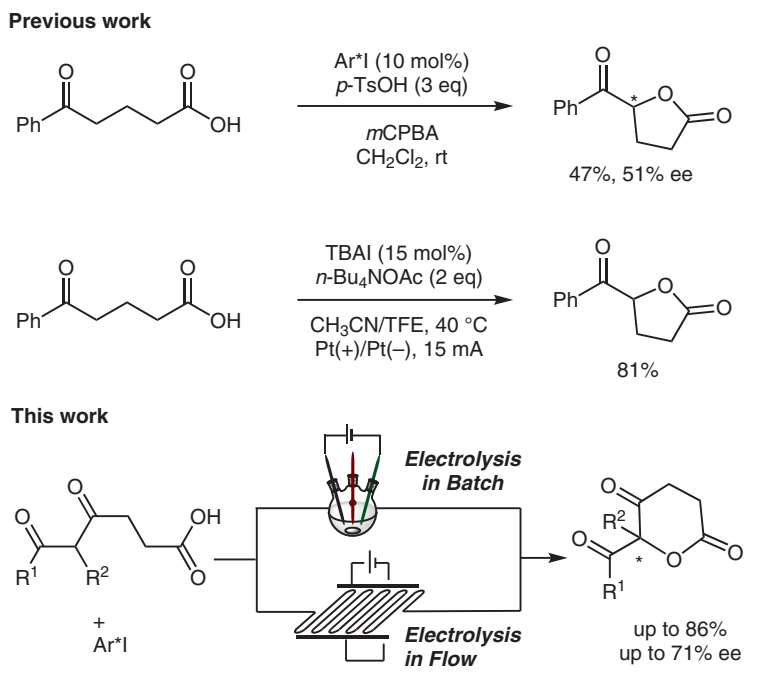

Scheme 1 Different methods for oxidative lactonization 
The anodic oxidation of chiral iodoarenes and subsequent enantioselective synthesis has never been reported, although there are many reported works on stereoselective oxidative functionalizations using hypervalent iodine reagents. ${ }^{15}$ Herein, we report the enantioselective electrochemical $\alpha$-lactonization and $\alpha$-alkoxylation of diketo acid derivatives with chiral iodoarenes as electron-transfer mediators. ${ }^{16}$ These asymmetric reactions have been performed in batch chemistry but can also proceed using an electrochemical flow reactor ${ }^{17}$ with lower amounts of electrolyte (Scheme 1, eq. 3).

Initial reactions were performed using diketo acid $\mathbf{1 a}$ as a model substrate for the enantioselective lactonization with chiral iodoarene $\mathbf{2 b}$ as redox mediator, and platinum as anode and cathode material, under galvanostatic conditions at $7 \mathrm{~mA}$ (Scheme 2). Since fluorinated solvents are known to stabilize iodine(III) reagents by the anodic oxidation of iodoarenes, ${ }^{14}$ 2,2,2-trifluoroethanol (TFE) was initially chosen as the solvent.

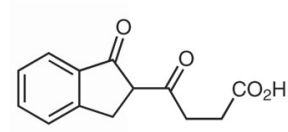

$1 a$

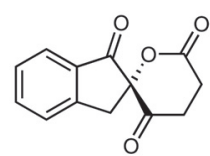

3a<smiles>[R7]C(=O)[C@@H](C)Oc1cc([R])cc(O[C@H](C)C([R])=O)c1I</smiles>

2a $\mathrm{R}^{1}=\mathrm{OMe}, \mathrm{R}^{2}=\mathrm{H}$

2b $\mathrm{R}^{1}=\mathrm{OMe}, \mathrm{R}^{2}=\mathrm{CO}_{2} \mathrm{Me}$

2c $\mathrm{R}^{1}=\mathrm{O} t-\mathrm{Bu}, \mathrm{R}^{2}=\mathrm{H}$

2d $R^{1}=O B n, R^{2}=H$

2e $R^{1}=\mathrm{NHPh}, \mathrm{R}^{2}=\mathrm{H}$

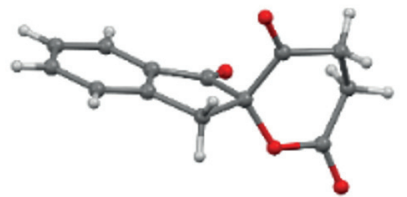

Scheme 2 Sample reaction for the development of electrochemical reaction conditions and X-ray structure of $(S)$-3a

Several commonly used electrolytes were investigated in the electrochemical reaction shown in Scheme 2 (Table 1, entries 1-3); the use of $n-\mathrm{Bu}_{4} \mathrm{NBF}_{4}$ as the electrolyte gave lactone 3a in good yield (70\%) and enantioselectivity (71\% ee) (entry 3 ). Although the reaction does proceed in the absence of trifluoroacetic acid (TFA), both the efficiency and enantioselectivity were decreased (Table 1 , entry 4 ). For this electrochemical process, the solvent HFIP is not a good choice and only results in moderate yield and lower enantioselectivity (Table 1, entry 5); no product was detected using acetonitrile as the solvent. There is also no product formed in the absence of either electrolyte or chiral iodoarene, and with catalytic amounts of $\mathbf{2} \mathbf{b}$ only traces of the product were observed (Table 1, entries 6-8). A decrease in the reaction temperature to $-10{ }^{\circ} \mathrm{C}$ did not improve the enantioselectivity, but only resulted in a lower yield of $3 \mathbf{a}$ (Table 1 , entry 9 ).
Table 1 Enantioselective Electrochemical Lactonization of $\mathbf{1 a}$ to $\mathbf{3 a}$ Using Chiral lodoarenes ${ }^{\mathrm{a}}$

\begin{tabular}{|c|c|c|c|}
\hline \multirow[t]{2}{*}{ Entry } & \multirow[t]{2}{*}{ Electrolyte } & \multicolumn{2}{|l|}{$3 a$} \\
\hline & & Yield (\%) & $\mathrm{ee}^{\mathrm{b}}(\%)$ \\
\hline 1 & $\mathbf{2 b}(1.2 \mathrm{eq}), \mathrm{LiClO}_{4}$ & 30 & 67 \\
\hline 2 & 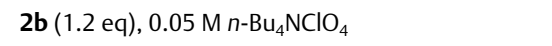 & 61 & 65 \\
\hline 3 & $2 \mathbf{b}(1.2 \mathrm{eq}), 0.05 \mathrm{M} \mathrm{n}-\mathrm{Bu}_{4} \mathrm{NBF}_{4}$ & 70 & 71 \\
\hline 4 & 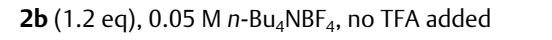 & 51 & 61 \\
\hline 5 & 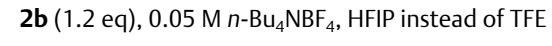 & 50 & 29 \\
\hline 6 & $\mathbf{2 b}(1.2 \mathrm{eq})$ & 0 & - \\
\hline 7 & no iodoarene, $0.05 \mathrm{M} \mathrm{LiClO}_{4}$ & 0 & - \\
\hline 8 & $2 \mathbf{b}(0.2 \mathrm{eq}), 0.05 \mathrm{M} \mathrm{n}-\mathrm{Bu}_{4} \mathrm{NBF}_{4}$ & $<5$ & - \\
\hline $9^{c}$ & $2 \mathbf{b}$ (1.2 eq), $0.05 \mathrm{M} \mathrm{n}-\mathrm{Bu}_{4} \mathrm{NBF}_{4}$ & 41 & 70 \\
\hline 10 & $2 \mathrm{a}(1.2 \mathrm{eq}), 0.05 \mathrm{M} \mathrm{n}-\mathrm{Bu}_{4} \mathrm{NBF}_{4}$ & 54 & 67 \\
\hline 11 & 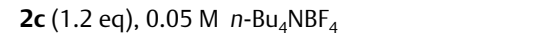 & 15 & 68 \\
\hline
\end{tabular}

a Reaction conditions: Pt cathode, Pt anode, 1 a $(0.025 \mathrm{M}), 2(0.03 \mathrm{M})$, electrolyte $(0.05 \mathrm{M})$, TFA $(0.075 \mathrm{M})$, solvent $(4 \mathrm{~mL})$, undivided cell (charge passed: $2.6 \mathrm{~F}$ )

${ }^{b}$ Determined by HPLC.

${ }^{\mathrm{C}}$ The reaction was performed at $-10^{\circ} \mathrm{C}$.

The absolute configuration of the major isomer of $\mathbf{3 a}$ was shown to be $(S)$ via X-ray crystallographic analysis (Scheme 2). ${ }^{18}$ Other lactate-based 2-iodoresorcinol derivatives were also employed as potential electron-transfer mediators, including iodoarenes that have previously found applications in the enantioselective oxidative dearomatization of naphthols. ${ }^{4}$ Neither $\mathbf{2 a}$ nor $\mathbf{2 c}$ gave better results than $\mathbf{2 b}$ (Table 1, entries 10 and 11) while the chiral iodoarenes $\mathbf{2 d}$ and $\mathbf{2 e}$ containing benzyl ester and amide functionalities decomposed after several minutes under the electrochemical reaction conditions. ${ }^{19}$

To explore the generality and the substrate scope of this electrochemical reaction, some diketo acid derivatives $\mathbf{1}$ were subjected to the electrolysis conditions (Scheme 3). The electrolysis of substrates bearing electron-rich or -poor groups on the indanone moiety gave the corresponding lactones $\mathbf{3 b}$-3d in moderate yields with reasonable enantioselectivities. For the naphthyl-substituted substrate 1e, the reaction proceeded in high yield leading to the product $3 \mathbf{e}$ in $63 \%$ ee. Also, tetralone derivatives such as 1f led to the cyclized product $3 \mathrm{f}$ in $58 \%$ yield and $47 \%$ ee, while lactone $\mathbf{3 g}$ without an aryl moiety was only formed in $36 \%$ yield as a racemate.

When the carbonyl group and carboxylic acid were both fixed to an aromatic ring as $\mathbf{3 h}$, the reaction proceeded well with good selectivity. Unfortunately, the attempted cyclization of the monocarbonyl substrate 5-oxo-5-phenylpentanoic acid (1i) failed to give any desired product $\mathbf{3 i}$, which was previously reported in high yield under electrochemical conditions with $n-\mathrm{Bu}_{4} \mathrm{NI}$ as the mediator. ${ }^{9}$ 

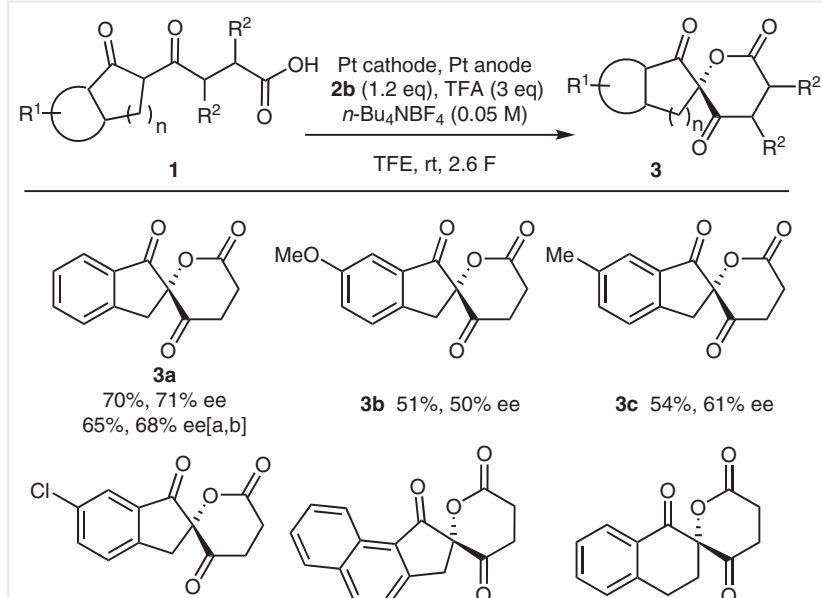

3b $51 \%, 50 \%$ ee 3c $54 \%, 61 \%$ ee
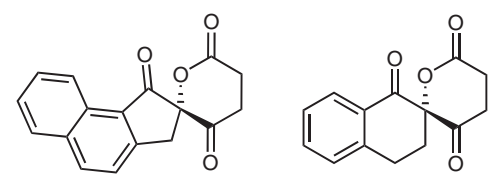

3d $40 \%, 68 \%$ ee 3e $87 \%, 63 \%$ ee

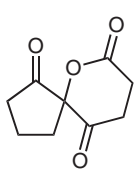

$3 \mathbf{g}$

$36 \%, 0 \%$ ee

$47 \%, 0 \%$ ee[b]

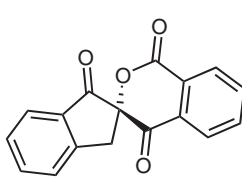

3h $64 \%, 67 \%$ ee

Scheme 3 Scope and limitations for enantioselective electrochemical spirolactonization using $\mathbf{2 b}$. Reagents and conditions: Pt cathode, Pt anode, 1 (0.025 M), $2 \mathbf{b}(0.03 \mathrm{M}), n-\mathrm{Bu}_{4} \mathrm{NBF}_{4}(0.05 \mathrm{M})$, TFA (0.075 M), TFE $(4 \mathrm{~mL})$, undivided cell (charge passed: $2.6 \mathrm{~F})$. [a] Current efficiency: 54\%. [b] Reagents and conditions: mCPBA (1.5 eq), 2 b (15 mol\%), TFA (3 eq), TFE (2 mL), rt, 24 h.

It has been reported that oxidative lactonization can be achieved with a combination of chiral iodoarenes and $m C P B A$ as stoichiometric oxidant. ${ }^{7}$ Therefore, the combination of $\mathbf{2} \mathbf{b}$ and $m C$ PBA was compared to the electrochemical reaction conditions, leading to products $\mathbf{3 a}, \mathbf{3 g}$ and $\mathbf{3 i}$. Compounds $\mathbf{3 a}$ and $\mathbf{3 g}$ were obtained with similar yields and selectivities, while $3 \mathbf{i}$ could be isolated in moderate yield and low enantioselectivity only using the combination of $\mathbf{2 b}$ and $m$ CPBA (Scheme 3 ).

With chiral iodoarene $\mathbf{2 b}$, intermolecular $\mathrm{C}-\mathrm{O}$ bond functionalization was also investigated using ester $\mathbf{4}$ as a substrate under the standard electrochemical conditions (Scheme 4). Protic solvents such as water, alcohols and acetic acid were chosen, together with TFE, due to their conductivity and nucleophilicity.

Pleasingly, the electrochemical reaction of ester $\mathbf{4}$ in a mixture of TFE $/ \mathrm{H}_{2} \mathrm{O}$ (3:1) with $\mathbf{2 b}$ as the chiral mediator gave the desired $\alpha$-hydroxy product $\mathbf{5 a}$ in moderate yield with $31 \%$ ee. Although the reaction efficiency was reduced in solvent mixtures of TFE with methanol or ethanol, high enantioselectivities (up to 79\% ee) were observed (5b and 5c). However, when the electrolysis was attempted in the solvent TFE/AcOH (3:1), the desired $\alpha$-acetoxy product $\mathbf{5 d}$ was not detected. The absolute configuration of compounds $\mathbf{5}$ was assigned by analogy to compound $\mathbf{3 a}$.

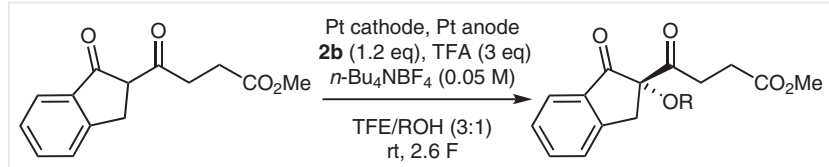

5a $\mathrm{R}=\mathrm{H}: \quad 66 \%, 31 \%$ ee 5b $\mathrm{R}=$ Me: $38 \%, 77 \%$ ee 5c $\mathrm{R}=\mathrm{Et}: \quad 40 \%, 79 \%$ ee 5d R=Ac: $0 \%$

Scheme 4 Intermolecular enantioselective electrosynthesis. Reagents and conditions: Pt cathode, Pt anode, $\mathbf{4}(0.1 \mathrm{mmol}), \mathbf{2 b}(0.12 \mathrm{mmol})$, $n-\mathrm{Bu}_{4} \mathrm{NBF}_{4}(0.05 \mathrm{M})$, TFA $(0.075 \mathrm{M}), \mathrm{TFE} / \mathrm{ROH}(3: 1 \mathrm{v} / \mathrm{v}, 4 \mathrm{~mL})$, undivided cell (charge passed: 2.6 F).

Compared with batch processes for electrochemical reactions, continuous flow reactors can reduce the amount or avoid the use of electrolytes because of the close distance of the electrodes. ${ }^{20}$ We have reported electrochemical flow microreactors for several oxidative transformations. ${ }^{17}$ Pleasingly, the present enantioselective lactonization could also be successfully accomplished using an electrochemical flow microreactor with a lower concentration of $n-\mathrm{Bu}_{4} \mathrm{NBF}_{4}$ $(5 \mathrm{mM})$ (Scheme 5), despite a slight decrease in the efficiency and enantioselectivity. There is no conversion without catalytic amounts of electrolyte and the conversion decreases when the platinum cathode is replaced by graphite. To the best of our knowledge, this is the first enantioselective reaction with iodine(III) reagents generated in an electrochemical flow microreactor.

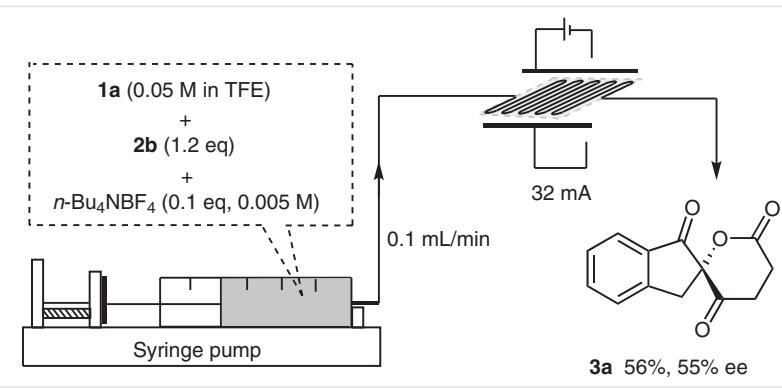

Scheme 5 Enantioselective electrochemical lactonization of $\mathbf{1 a}$ to $\mathbf{3 a}$ in a flow microreactor

Additional experiments were carried out to further explore the mechanism of this enantioselective electrolytic lactonization. Initially, a stepwise batch process was performed. After the anodic oxidation of mediator $\mathbf{2} \mathbf{b}$, substrate $\mathbf{1 a}$ was added to the reaction mixture. However, this stepwise protocol only led to the desired product 3a in $9 \%$ yield (65\% ee) (Scheme 6 ). In the ${ }^{1} \mathrm{H}$ NMR spectra of the electrolyzed $\mathbf{2 b}$ in TFE, a downfield peak at around 7.24 ppm was observed indicating the formation of an iodine(III) species, but this peak disappeared after several hours or after removal of the solvent (see the Supporting Information). This indicates the formation of an unstable iodine(III) species $\mathrm{Ar}^{*}-\mathrm{IL}_{2}$ by electrolysis of iodoarene $\mathbf{2 b}$. In addition, cy- 
clic voltammetry in TFE showed a lower potential $(1.83 \mathrm{~V}$, vs $\mathrm{Ag} / \mathrm{AgCl})$ for $\mathbf{2 b}$ than for $\mathbf{1 a}(2.07 \mathrm{~V}$, vs $\mathrm{Ag} / \mathrm{AgCl})$ indicating that $\mathbf{2 b}$ is easier oxidized than $\mathbf{1 a}$ in the one-pot electrolysis (Figure 1). ${ }^{8 c}$

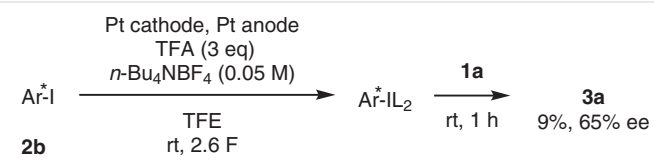

Scheme $\mathbf{6}$ Electrolysis of $\mathbf{2} \mathbf{b}$ and reaction with $\mathbf{1} \mathbf{a}$ in a stepwise process

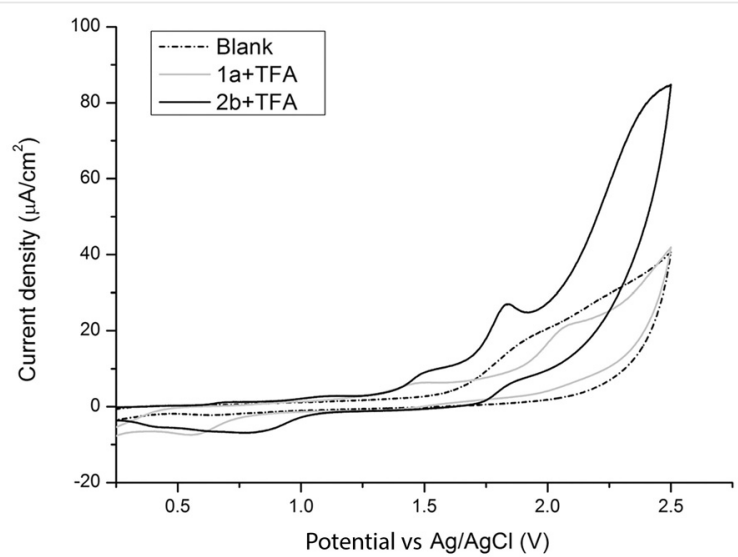

Figure 1 Cyclic voltammograms using $n-\mathrm{Bu}_{4} \mathrm{NBF}_{4}(0.1 \mathrm{M})$ as electrolyte in TFE at $20 \mathrm{mV} \mathrm{s}^{-1}$, under $\mathrm{N}_{2}$. Working electrode: glass carbon; reference electrode: $\mathrm{Ag} / \mathrm{AgCl}$ in $3 \mathrm{M} \mathrm{NaCl}$; auxiliary electrode: Pt wire. Blank (dots); $\mathbf{1} \mathbf{a}$ with 3 eq of TFA (gray); $\mathbf{2} \mathbf{b}$ with 3 eq of TFA (black).

Based on the investigations from Muñiz, Ishihara and co-workers, ${ }^{3 e}$ two structures for a possible explanation of the observed stereoselectivities are shown in Figure 2. These structures show the intermediates after the reaction of the substrate enolates with the iodine(III) reagent. Due to the steric hindrance between the indanone moiety and the lactate ester, intramolecular cyclization favorably proceeds through intermediate 6 rather than through intermediate $\mathbf{7}$.

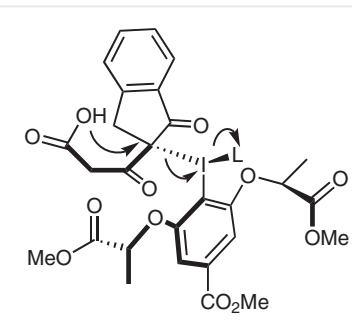

6

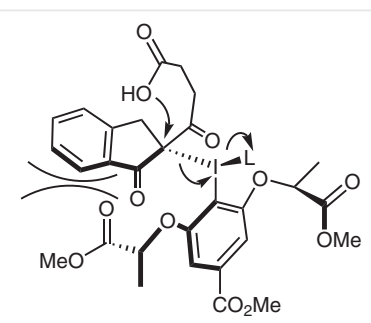

7
Figure 2 Proposed intermediates in the stereoselective lactonization of $3 a$
In summary, we have developed an electrochemical method for enantioselective lactonization using chiral lactate-based iodoarenes as redox mediators. This protocol was also applied to the intermolecular $\alpha$-alkoxylation of diketo esters with good enantioselectivities. Furthermore, it was demonstrated that this enantioselective transformation could be adapted to an electrochemical flow microreactor with lower supporting electrolyte concentration. Further studies on stereoselective electrosynthesis using chiral iodoarenes are currently in progress.

\begin{abstract}
All reactions were carried out in oven-dried glassware under an atmosphere of nitrogen/argon using anhydrous solvents. All commercial reagents were used as received. ${ }^{1} \mathrm{H}$ NMR spectra were recorded at 400 or $500 \mathrm{MHz}$ on Bruker DPX 400 or DPX 500 spectrometers. Chemical shifts are reported in parts per million $(\mathrm{ppm}, \delta)$ relative to TMS $(\delta 0.00) .{ }^{1} \mathrm{H}$ NMR splitting patterns are designated as singlet $(\mathrm{s})$, doublet $(\mathrm{d})$, doublet of doublet $(\mathrm{dd})$, triplet $(\mathrm{t})$, quartet $(\mathrm{q})$, multiplet (m). ${ }^{13} \mathrm{C}$ NMR spectra were recorded at 100 or $125 \mathrm{MHz}$. Mass spectra were obtained using a Waters Xevo G2-S ESI mass spectrometers. IR spectra were recorded as neat on a Shimadzu FTIR Affinity-1S spectrophotometer. Melting points were determined using a Gallenkamp hot-stage apparatus and are uncorrected. Optical rotations were measured using a $10.0 \mathrm{~mL}$ cell with a $1.0 \mathrm{dm}$ path length on a SCHMIDT + HAENSCH UniPol L polarimeter apparatus and are reported as $[\alpha]$ ( $c$ in g per $100 \mathrm{~mL}$, solvent) at $20^{\circ} \mathrm{C}$.
\end{abstract}

Methyl 4-0xo-4-(1-oxo-2,3-dihydro-1H-inden-2-yl)butanoate (4) To a solution of diisopropylamine $(1.6 \mathrm{~g}, 15.8 \mathrm{mmol})$ in THF $(40 \mathrm{~mL})$ was added $2.5 \mathrm{M} n$-BuLi in hexane $(6.9 \mathrm{~mL}, 17.4 \mathrm{mmol})$ at $-78^{\circ} \mathrm{C}$. The resulting solution was stirred at $-78{ }^{\circ} \mathrm{C}$ for $30 \mathrm{~min}$ and then at room temperature for an additional $30 \mathrm{~min}$. The solution was cooled to $-78{ }^{\circ} \mathrm{C}$, and to this solution was added dropwise a solution of 1-indanone $(1 \mathrm{~g}, 8 \mathrm{mmol})$ in THF $(10 \mathrm{~mL})$. After $1 \mathrm{~h}$ at $-78^{\circ} \mathrm{C}$, to the solution was added dropwise methyl-4-chloro-4-oxobutyrate $(1.36 \mathrm{~mL}, 11$ $\mathrm{mmol})$ in THF ( $5 \mathrm{~mL})$. The resulting solution was warmed to room temperature over $2 \mathrm{~h}$, and then quenched with saturated $\mathrm{NH}_{4} \mathrm{Cl}$ solution. The organic phase was separated, and the water phase was extracted with $\mathrm{Et}_{2} \mathrm{O}(3 \times 20 \mathrm{~mL})$. The combined organic layers were dried, concentrated and purified by chromatography (petroleum ether/EtOAc, 5:1) to give 4 as a colorless solid; yield: $1.01 \mathrm{~g} \mathrm{(51 \% );} \mathrm{mp}$ $57-59{ }^{\circ} \mathrm{C}$.

IR (neat): 3025, 1734, 1628, 1545, 1221, 1167, 1038, 779, $731 \mathrm{~cm}^{-1}$.

${ }^{1} \mathrm{H} \mathrm{NMR}\left(500 \mathrm{MHz}, \mathrm{CDCl}_{3}\right): \delta=7.78(\mathrm{~d}, J=8.0 \mathrm{~Hz}, 1 \mathrm{H}), 7.55-7.48(\mathrm{~m}, 2$ $\mathrm{H}), 7.41(\mathrm{t}, J=7.5 \mathrm{~Hz}, 1 \mathrm{H}), 3.70(\mathrm{~s}, 3 \mathrm{H}), 3.64(\mathrm{~s}, 2 \mathrm{H}), 2.82(\mathrm{t}, J=6.0 \mathrm{~Hz}$, $2 \mathrm{H}), 2.75(\mathrm{t}, J=6.0 \mathrm{~Hz}, 2 \mathrm{H})$.

${ }^{13} \mathrm{C}$ NMR $\left(125 \mathrm{MHz}, \mathrm{CDCl}_{3}\right): \delta=187.5,182.4,173.0,146.8,137.7$, 132.4, 127.3, 125.6, 122.8, 110.6, 51.9, 30.5, 30.4, 28.9.

HRMS (ESI): $m / z$ calcd for $\mathrm{C}_{14} \mathrm{H}_{15} \mathrm{O}_{4}[\mathrm{M}+\mathrm{H}]^{+}$: 247.0965; found: 247.0968.

\section{4-0xo-4-(1-oxo-2,3-dihydro-1H-inden-2-yl)butanoic Acid (1a)}

Compound 4 (1.2 g, $5 \mathrm{mmol})$ was dissolved in $\mathrm{THF} / \mathrm{MeOH} /$ water $(3: 1: 1 \mathrm{v} / \mathrm{v} / \mathrm{v}, 20 \mathrm{~mL})$. To this mixture $1 \mathrm{M} \mathrm{LiOH}$ in water $(10 \mathrm{~mL})$ was added and the resulting solution stirred overnight. After removal of organic solvent in vacuo, the aqueous phase was acidified with $\mathrm{HCl}$ until $\mathrm{pH}$ 3. The mixture was extracted with EtOAc $(3 \times 20 \mathrm{~mL})$. The combined organic fractions were dried with sodium sulfate and con- 
centrated in vacuo to give the crude acid 1a, which was recrystallized from EtOAc to give 1a as a white solid; yield: $1.03 \mathrm{~g}$ (89\%); mp 119$121^{\circ} \mathrm{C}$.

IR (neat): 3025, 1699, 1624, 1549, 1404, 1067, 972, $775 \mathrm{~cm}^{-1}$.

${ }^{1} \mathrm{H}$ NMR $\left(500 \mathrm{MHz}, \mathrm{CD}_{3} \mathrm{OD}\right): \delta$ (two isomers) $=7.70(\mathrm{~d}, J=7.5 \mathrm{~Hz}, 0.77$ $\mathrm{H})$ (major), 7.68-7.60 (m, 1 H), 7.57-7.52 (m, $2 \mathrm{H}), 7.44-7.35(\mathrm{~m}, 1.23$ $\mathrm{H}), 3.66$ (s, $1.54 \mathrm{H}$ ) (major), $3.60(\mathrm{~d}, J=16.5 \mathrm{~Hz}, 0.46 \mathrm{H}), 3.28-3.25(\mathrm{~m}$, $0.23 \mathrm{H}), 3.19(\mathrm{~d}, J=16.5 \mathrm{~Hz}, 0.46 \mathrm{H}), 2.98-2.90(\mathrm{~m}, 0.46 \mathrm{H}), 2.83(\mathrm{t}$, $J=6.5 \mathrm{~Hz}, 1.54 \mathrm{H}$ ) (major), 2.70 (t, $J=6.5 \mathrm{~Hz}, 1.63 \mathrm{H}$ ) (major), 2.63$2.50(\mathrm{~m}, 0.77 \mathrm{H})$.

${ }^{13} \mathrm{C}$ NMR $\left(125 \mathrm{MHz}, \mathrm{CD}_{3} \mathrm{OD}\right): \delta=204.4,202.1,176.3,156.2,148.7$, $139.1,136.8,136.5,133.7,128.9,128.5,128.0,127.0,125.3,123.6$, $112.3,38.6,31.6,31.5,29.9,29.3,28.8$.

HRMS (ESI): $m / z$ calcd for $\mathrm{C}_{13} \mathrm{H}_{11} \mathrm{O}_{4}[\mathrm{M}-\mathrm{H}]^{-}:$:231.0663; found: 231.0665 .

\section{4-(6-Methoxy-1-oxo-2,3-dihydro-1H-inden-2-yl)-4-oxobutanoic Acid (1b)}

Yield: $406 \mathrm{mg}$ (31\%); white solid; mp 132-134 ${ }^{\circ} \mathrm{C}$.

IR (neat): 3003, 1718, 1647, 1576, 1492, 1375, 1024, 877, $786 \mathrm{~cm}^{-1}$.

${ }^{1} \mathrm{H}$ NMR $\left(400 \mathrm{MHz}\right.$, DMSO- $\left.d_{6}\right): \delta$ (two isomers) $=12.2($ br s, $1 \mathrm{H}), 7.52$ $(\mathrm{d}, J=8.4 \mathrm{~Hz}, 0.40 \mathrm{H}), 7.48$ (d, $J=8.4 \mathrm{~Hz}, 0.60 \mathrm{H}$ ) (major), 7.29 (dd, $J=8.4,2.8 \mathrm{~Hz}, 0.40 \mathrm{H}$ ), 7.25-7.20 (m, $0.60 \mathrm{H}$ ) (major), 7.15 (dd, $J=8.4$, $2.8 \mathrm{~Hz}, 0.60 \mathrm{H}$ ) (major), $7.09(\mathrm{~d}, J=2.8 \mathrm{~Hz}, 0.40 \mathrm{H}), 4.25$ (dd, $J=8.0,2.8$ $\mathrm{Hz}, 0.60 \mathrm{H}$ ) (major), 3.81 (s, $2 \mathrm{H}), 3.79$ (s, $1 \mathrm{H}), 3.56$ (br s, $1.40 \mathrm{H}), 3.37$ (dd, $J=13.2,2.8 \mathrm{~Hz}, 0.60 \mathrm{H}$ ) (major), 3.20-3.07 (m, $1 \mathrm{H}), 2.92-2.80(\mathrm{~m}$, $1.60 \mathrm{H}$ ) (major), $2.56(\mathrm{t}, J=2.8 \mathrm{~Hz}, 1.40 \mathrm{H}), 2.43(\mathrm{t}, J=6.8 \mathrm{~Hz}, 0.60 \mathrm{H})$ (major).

${ }^{13} \mathrm{C}$ NMR $\left(100 \mathrm{MHz}\right.$, DMSO- $\left.d_{6}\right): \delta=202.8,199.8,173.7,173.6,159.2$, 158.9, 147.0, 136.1, 127.7, 126.6, 124.3, 119.6, 112.5, 105.4, 105.1, 61.4, 55.6, 55.4, 37.1, 31.6, 30.1, 28.4, 27.6, 27.4.

HRMS (ESI): $m / z$ calcd for $\mathrm{C}_{14} \mathrm{H}_{13} \mathrm{O}_{5}[\mathrm{M}-\mathrm{H}]^{-}:$261.0768; found: 261.0770 .

\section{4-(6-Methyl-1-oxo-2,3-dihydro-1H-inden-2-yl)-4-oxobutanoic Acid (1c)}

Yield: 677 mg (55\%); white solid; mp $138-140{ }^{\circ} \mathrm{C}$.

IR (neat): 2950, 1703, 1614, 1544, 1207, 1165, 1111, $1031 \mathrm{~cm}^{-1}$

${ }^{1} \mathrm{H}$ NMR $\left(400 \mathrm{MHz}\right.$, DMSO- $\left.d_{6}\right): \delta$ (two isomers) $=12.1$ (br s, $\left.1 \mathrm{H}\right)$, 7.57-7.37 (m, $3 \mathrm{H}), 4.23(\mathrm{dd}, J=8.0,3.2 \mathrm{~Hz}, 0.40 \mathrm{H}), 3.60(\mathrm{~s}, 1.3 \mathrm{H})$ (major), 3.43 (dd, $J=17.6,3.2 \mathrm{~Hz}, 0.40 \mathrm{H}), 3.24-3.10(\mathrm{~m}, 1 \mathrm{H}), 2.95-$ $2.75(\mathrm{~m}, 1.60 \mathrm{H})$ (major), $2.57(\mathrm{t}, J=6.4 \mathrm{~Hz}, 1.40 \mathrm{H}), 2.44(\mathrm{t}, J=6.4 \mathrm{~Hz}$, $1 \mathrm{H}), 2.39(\mathrm{~m}, 3 \mathrm{H})$.

${ }^{13} \mathrm{C}$ NMR (100 MHz, DMSO- $\left.d_{6}\right)$ : $\delta=202.9,199.9,173.7,173.6,151.7$, 137.3, 136.6, 135.0, 126.5, 125.5, 123.6, 122.0, 61.0, 37.1, 31.5, 30.4, 27.7, 27.6, 20.9, 20.5.

HRMS (ESI): $m / z$ calcd for $\mathrm{C}_{14} \mathrm{H}_{13} \mathrm{O}_{4}[\mathrm{M}-\mathrm{H}]^{-}:$245.0819; found: 245.0822 .

\section{4-(6-Chloro-1-oxo-2,3-dihydro-1H-inden-2-yl)-4-oxobutanoic Acid (1d)}

Yield: $373 \mathrm{mg}$ (28\%); white solid; $\mathrm{mp} 134-136{ }^{\circ} \mathrm{C}$.

IR (neat): 2950, 1701, 1626, 1541, 1217, 1078, 813, $748 \mathrm{~cm}^{-1}$.
${ }^{1} \mathrm{H}$ NMR (500 MHz, $\mathrm{CD}_{3} \mathrm{OD}$ ): $\delta$ (two isomers) $=7.70-7.63(\mathrm{~m}, 3 \mathrm{H}$ ), $3.67(\mathrm{~s}, 1.50 \mathrm{H})$ (major), $3.60(\mathrm{~d}, J=18.0 \mathrm{~Hz}, 0.30 \mathrm{H}), 3.30-3.24(\mathrm{~m}$, $0.30 \mathrm{H}), 3.18$ (d, $J=18 \mathrm{~Hz}, 0.30 \mathrm{H}), 3.00-2.90(\mathrm{~m}, 0.30 \mathrm{H}), 2.90-2.82$ $(\mathrm{m}, 1.50 \mathrm{H})$ (major), $2.71(\mathrm{t}, J=7.0 \mathrm{~Hz}, 1.50 \mathrm{H})$ (major), 2.60-2.54 (m, $0.30 \mathrm{H})$.

${ }^{13} \mathrm{C}$ NMR $\left(125 \mathrm{MHz}, \mathrm{CD}_{3} \mathrm{OD}\right): \delta=203.9,200.7,176.4,176.3,154.4$, $146.4,140.8,138.2,136.5,134.7,133.2,129.5,128.4,124.7,123.1$, $113.3,38.5,32.3,31.6,29.7,28.9,28.8$.

HRMS (ESI): $m / z$ calcd for $\mathrm{C}_{13} \mathrm{H}_{10} \mathrm{ClO}_{4}[\mathrm{M}-\mathrm{H}]^{-}:$: 265.0273, 267.0243; found: $265.0273,267.0244$.

\section{4-0xo-4-(1-oxo-2,3-dihydro-1H-cyclopenta[a]naphthalen-2-} yl)butanoic Acid (1e)

Yield: $1.01 \mathrm{~g}(71 \%)$; white solid; $\mathrm{mp} 164-166{ }^{\circ} \mathrm{C}$.

IR (neat): 3010, 1710, 1591, 1541, 1340, 1219, 1062, 870, $783 \mathrm{~cm}^{-1}$. ${ }^{1} \mathrm{H}$ NMR (500 MHz, DMSO- $d_{6}$ ): $\delta$ (two isomers) $=12.2$ (br s, $1 \mathrm{H}$ ), 8.97 $(\mathrm{d}, J=8.5 \mathrm{~Hz}, 0.45 \mathrm{H}), 8.92(\mathrm{~d}, J=8.5 \mathrm{~Hz}, 0.55 \mathrm{H}$ ) (major), 8.27 (d, $J=7.0 \mathrm{~Hz}, 0.55 \mathrm{H}$ ) (major), $8.20(\mathrm{~d}, J=7.5 \mathrm{~Hz}, 0.45 \mathrm{H}), 8.08$ (d, $J=8.0$ $\mathrm{Hz}, 1 \mathrm{H}), 7.77-7.67$ (m, $2 \mathrm{H}), 7.63$ (t, $J=7.5 \mathrm{~Hz}, 1 \mathrm{H}), 4.34$ (tt, $J=7.5$, $2.5 \mathrm{~Hz}, 0.45 \mathrm{H}), 3.59-3.52(\mathrm{~m}, 0.55 \mathrm{H})$ (major), 3.35-3.16 (m, $1.45 \mathrm{H})$, $3.00-2.90(\mathrm{~m}, 0.55 \mathrm{H})$ (major), $2.78(\mathrm{t}, J=7.5 \mathrm{~Hz}, 1 \mathrm{H}), 2.48-2.44(\mathrm{~m}, 2$ $\mathrm{H})$.

${ }^{13} \mathrm{C}$ NMR $\left(125 \mathrm{MHz}\right.$, DMSO- $\left.d_{6}\right): \delta=203.1,200.4,190.6,173.5,173.4$, $158.4,149.7,136.5,133.8,132.3,131.2,128.7,128.2,126.5,124.3$, 123.8, 123.2, 122.7, 111.0, 61.1, 37.1, 30.3, 29.6, 29.0, 28.6, 27.6.

HRMS (ESI): $m / z$ calcd for $\mathrm{C}_{17} \mathrm{H}_{13} \mathrm{O}_{4}[\mathrm{M}-\mathrm{H}]^{-}$: 281.0819; found: 281.0820 .

\section{4-0xo-4-(1-oxo-1,2,3,4-tetrahydronaphthalen-2-yl)butanoic Acid} (1f)

Yield: $627 \mathrm{mg}$ (51\%); white solid; $\mathrm{mp} 102-104{ }^{\circ} \mathrm{C}$.

IR (neat): 2934, 1697, 1614, 1558, 1410, 1153, 935, 781, $737 \mathrm{~cm}^{-1}$. ${ }^{1} \mathrm{H}$ NMR $\left(500 \mathrm{MHz}, \mathrm{CDCl}_{3}\right.$ ): $\delta$ (two isomers) $=8.02$ (dd, $J=8.0,1.0 \mathrm{~Hz}$, $0.10 \mathrm{H}$ ), $7.90(\mathrm{dd}, J=8.0,1.0 \mathrm{~Hz}, 0.90 \mathrm{H}$ ) (major), $7.49(\mathrm{td}, J=9.0,1.5$ $\mathrm{Hz}, 0.10 \mathrm{H}$ ), 7.38 (td, $J=7.5,1.5 \mathrm{~Hz}, 0.90 \mathrm{H})$ (major), 7.34-7.28 (m, 1 H), $7.25(\mathrm{~d}, J=7.5 \mathrm{~Hz}, 0.10 \mathrm{H}), 7.19(\mathrm{~d}, J=7.5 \mathrm{~Hz}, 0.90 \mathrm{H}$ ) (major), 2.92-2.84 (m, $4 \mathrm{H}), 2.75(\mathrm{t}, J=6.5 \mathrm{~Hz}, 2 \mathrm{H}), 2.67-2.62(\mathrm{~m}, 2 \mathrm{H})$.

${ }^{13} \mathrm{C}$ NMR $\left(125 \mathrm{MHz}, \mathrm{CDCl}_{3}\right): \delta=197.4,178.3,173.0,140.3,131.7$, $130.4,127.5,126.8,125.6,105.5,31.9,28.2,28.0,21.8$.

HRMS (ESI): $m / z$ calcd for $\mathrm{C}_{14} \mathrm{H}_{13} \mathrm{O}_{4}[\mathrm{M}-\mathrm{H}]^{-}:$: 245.0819; found: 245.0821.

\section{2-(1-0xo-2,3-dihydro-1H-inden-2-ylcarbonyl)benzoic Acid (1h)}

Yield: $1.08 \mathrm{~g}(77 \%)$; white solid; $\mathrm{mp} 142-144^{\circ} \mathrm{C}$.

IR (neat): 2893, 1699, 1647, 1568, 1361, 1205, 1091, 999, 849, 781 $\mathrm{cm}^{-1}$.

${ }^{1} \mathrm{H} \mathrm{NMR}\left(500 \mathrm{MHz}, \mathrm{CD}_{3} \mathrm{OD}\right): \delta$ (two isomers) $=8.00(\mathrm{dd}, J=7.5,1.0 \mathrm{~Hz}$, $0.5 \mathrm{H}$ ), 7.82 (br s, $0.5 \mathrm{H}$ ), 7.76 (d, $J=8.0 \mathrm{~Hz}, 0.55 \mathrm{H}$ ) (major), 7.74-7.52 $(\mathrm{m}, 5 \mathrm{H}), 7.48(\mathrm{~d}, J=7.5 \mathrm{~Hz}, 0.55 \mathrm{H})($ major $), 7.43(\mathrm{t}, J=7.5 \mathrm{~Hz}, 0.55 \mathrm{H})$ (major), 7.36 (t, $J=8.0 \mathrm{~Hz}, 0.55 \mathrm{H}$ ) (major), 3.46-3.15 (m, $2 \mathrm{H}$ ).

${ }^{13} \mathrm{C}$ NMR $\left(125 \mathrm{MHz}, \mathrm{CD}_{3} \mathrm{OD}\right): \delta=189.7,169.8,155.4,139.1,138.7$, 136.7, 134.0, 133.4, 131.1, 129.8, 128.6, 127.9, 127.0, 124.8, 123.7, $112.8,32.4,30.5$.

HRMS (ESI): $m / z$ calcd for $\mathrm{C}_{17} \mathrm{H}_{11} \mathrm{O}_{4}[\mathrm{M}-\mathrm{H}]^{-}:$:279.0663; found: 279.0665 . 


\section{4-0xo-4-(2-oxocyclopentyl)butanoic Acid (1g)}

A mixture of cyclopentanone $(2.6 \mathrm{~mL}, 30 \mathrm{mmol})$ and pyrrolidine $(3.0$ $\mathrm{mL}, 36 \mathrm{mmol})$ in benzene $(12 \mathrm{~mL})$ was refluxed using a Dean-Stark apparatus overnight. The solvents and excess pyrrolidine were removed in vacuo. The crude enamine and dry triethylamine $(4.6 \mathrm{~mL}$, $33 \mathrm{mmol}$ ) were dissolved in benzene $(10 \mathrm{~mL})$, and to this solution was added dropwise methyl-4-chloro-4-oxobutyrate $(3 \mathrm{~mL}, 33 \mathrm{mmol}$ ). The reaction mixture was then heated at reflux for $8 \mathrm{~h}$, cooled to room temperature and filtered through Celite. The filtrate was concentrated in vacuo to give the acylated enamine which was used without further purification. The acylated enamine was dissolved in water $(7.5 \mathrm{~mL})$, acetic acid $(7.5 \mathrm{~mL})$ and THF $(15 \mathrm{~mL})$, and the resultant dark-brown solution stirred at room temperature for $24 \mathrm{~h}$. The reaction mixture was then added to water $(20 \mathrm{~mL})$ and chloroform (50 $\mathrm{mL}$ ), the phases were separated, and the aqueous phase was extracted with chloroform. The combined organic extracts were dried, the solvent was removed and the remainder was purified by chromatography using petroleum ether/ethyl acetate $4: 1$ as eluent to give $\mathbf{1 g}$ as a yellow oil; yield: $2.85 \mathrm{~g}$ (48\%).

IR (neat): 2981, 2864, 1697, 1631, 1589, 1240, $928 \mathrm{~cm}^{-1}$.

${ }^{1} \mathrm{H}$ NMR $\left(500 \mathrm{MHz}, \mathrm{CDCl}_{3}\right): \delta$ (two isomers) $=3.40(\mathrm{t}, J=8.0 \mathrm{~Hz}, 1 \mathrm{H}$ ), 3.18 (dd, $J=7.0,5.5 \mathrm{~Hz}, 0.44 \mathrm{H}$ ), 3.14 (dd, $J=7.0,5.5 \mathrm{~Hz}, 0.55 \mathrm{H}$ ) (major), 2.84-2.75 (m, $1 \mathrm{H}), 2.73-2.65(\mathrm{~m}, 2.7 \mathrm{H})$ (major), 2.63-2.54 (m, 4 $\mathrm{H}), 2.50-2.39(\mathrm{~m}, 2.7 \mathrm{H})$ (major), 2.37-2.20 (m, $2.44 \mathrm{H}), 2.12-2.00(\mathrm{~m}$, $2 \mathrm{H}), 1.96-1.80(\mathrm{~m}, 2.55 \mathrm{H})$ (major).

${ }^{13} \mathrm{C}$ NMR $\left(125 \mathrm{MHz}, \mathrm{CDCl}_{3}\right): \delta=212.8,202.5,199.5,181.5,178.0$, 109.8, 61.9, 38.7, 37.2, 35.8, 30.1, 28.6, 27.7, 25.8, 25.1, 20.7, 20.1.

HRMS (ESI): $m / z$ calcd for $\mathrm{C}_{9} \mathrm{H}_{11} \mathrm{O}_{4}[\mathrm{M}-\mathrm{H}]^{-}$: 183.0663; found: 183.0666.

\section{Dimethyl (2R,2'R)-2,2'-((2-Iodo-5-(methoxycarbonyl)-1,3-phenyl-} ene)bis(oxy))dipropionate (2b)

Diisopropyl azodicarboxylate (10 mL, $50 \mathrm{mmol}, 2.5 \mathrm{eq})$ was added dropwise via syringe over $30 \mathrm{~min}$ to a stirred suspension of methyl 3,5-dihydroxy-4-iodobenzoate (5.9 g, $20.0 \mathrm{mmol}$ ), triphenylphosphine (13 g, $50 \mathrm{mmol}, 2.5 \mathrm{eq})$ and methyl $(S)-(-)$-lactate $(4.85 \mathrm{~mL}, 50$ $\mathrm{mmol}, 2.5 \mathrm{eq})$ in THF $(100 \mathrm{~mL})$ at $0{ }^{\circ} \mathrm{C}$. The reaction mixture was warmed to room temperature and stirred overnight. The resultant residue was purified by column chromatography (petroleum ether/EtOAc, 3:1) to give $\mathbf{2 b}$ as a white solid; yield: $6.62 \mathrm{~g}$ (71\%); mp 92-94 ${ }^{\circ} \mathrm{C}$.

$[\alpha]_{\mathrm{D}}^{20}+11\left(c 0.73, \mathrm{CHCl}_{3}\right)$.

IR (neat): 2949, 1743, 1712, 1573, 1417, 1240, 1132, 1072, 1008, 966, $854 \mathrm{~cm}^{-1}$.

${ }^{1} \mathrm{H}$ NMR $\left(500 \mathrm{MHz}, \mathrm{CDCl}_{3}\right): \delta=7.01(\mathrm{~s}, 2 \mathrm{H}), 4.88(\mathrm{q}, J=7.0 \mathrm{~Hz}, 2 \mathrm{H})$, $3.88(\mathrm{~s}, 3 \mathrm{H}), 3.76(\mathrm{~s}, 6 \mathrm{H}), 1.71(\mathrm{~d}, J=7.0 \mathrm{~Hz}, 6 \mathrm{H})$.

${ }^{13} \mathrm{C}$ NMR $\left(125 \mathrm{MHz}, \mathrm{CDCl}_{3}\right): \delta=171.6,166.1,158.2,131.7,107.2,87.3$, $74.1,55.4,18.5$.

HRMS (ESI): $m / z$ calcd for $\mathrm{C}_{16} \mathrm{H}_{20} \mathrm{IO}_{8}[\mathrm{M}+\mathrm{H}]^{+}$: 467.0197; found: 467.0194.

(S)-4',5'-Dihydrospiro[indene-2,2'-pyran]-1,3',6'(3H)-trione (3a); Typical Procedure for the Electrochemical Lactonization of 1

A 10-mL three-necked round-bottomed flask was equipped with a magnetic stirrer, and platinum plate $\left(1 \mathrm{~cm}^{2}\right)$ electrode as the working electrode and counter electrode. The substrate $1 \mathrm{a}$ ( $23 \mathrm{mg}, 0.1 \mathrm{mmol})$, chiral iodobenzene $\mathbf{2 b}$ (56 mg, $0.12 \mathrm{mmol}$ ), TFA (34 mg, $0.3 \mathrm{mmol}$ ) and supporting electrolyte $n-\mathrm{Bu}_{4} \mathrm{NBF}_{4}(66 \mathrm{mg}, 0.2 \mathrm{mmol})$ were added to the solvent TFE $(4 \mathrm{~mL})$. The resulting mixture was stirred and electrolyzed under galvanostatic conditions $\left(7 \mathrm{~mA} / \mathrm{cm}^{2}\right)$ at room temperature for $1 \mathrm{~h}$. The solvent was removed in vacuo and the residue purified by column chromatography (petroleum ether/EtOAc, $3: 1$ ) to give 3a as a white solid; yield: $16 \mathrm{mg}(70 \%)$; $\mathrm{mp} 162-164{ }^{\circ} \mathrm{C} ; 71 \%$ ee (determined by HPLC).

$[\alpha]_{\mathrm{D}}^{20}+27\left(\right.$ c $\left.0.4, \mathrm{CHCl}_{3}\right)$.

IR (neat): 2922, 1749, 1707, 1606, 1411, 1271, 1215, 1090, 972, 768 $\mathrm{cm}^{-1}$.

${ }^{1} \mathrm{H}$ NMR $\left(500 \mathrm{MHz}, \mathrm{CDCl}_{3}\right): \delta=7.75(\mathrm{~d}, J=7.5 \mathrm{~Hz}, 1 \mathrm{H}), 7.70(\mathrm{t}, J=6.0$ $\mathrm{Hz}, 1 \mathrm{H}), 7.51(\mathrm{~d}, J=8.0 \mathrm{~Hz}, 1 \mathrm{H}), 7.44(\mathrm{t}, J=7.0 \mathrm{~Hz}, 1 \mathrm{H}), 3.77(\mathrm{~d}$, $J=17.0 \mathrm{~Hz}, 1 \mathrm{H}), 3.73-3.63(\mathrm{~m}, 1 \mathrm{H}), 3.32(\mathrm{~d}, J=17.0 \mathrm{~Hz}, 1 \mathrm{H}), 3.01(\mathrm{dt}$, $J=17.5,4.0 \mathrm{~Hz}, 1 \mathrm{H}), 2.83(\mathrm{dt}, J=10.0,2.5 \mathrm{~Hz}, 2 \mathrm{H})$.

${ }^{13} \mathrm{C}$ NMR $\left(125 \mathrm{MHz}, \mathrm{CDCl}_{3}\right): \delta=201.8,197.3,169.0,151.9,137.0$, 132.4, 128.6, 126.2, 125.7, 92.7, 38.6, 33.4, 27.1.

HRMS (ESI): $m / z$ calcd for $\mathrm{C}_{13} \mathrm{H}_{11} \mathrm{O}_{4}[\mathrm{M}+\mathrm{H}]^{+}$: 231.0652; found: 231.0653.

HPLC (YMC Chiral Amylose-C S-5 $\mu \mathrm{m}(25 \mathrm{~cm})$, hexane/i-PrOH, 90:10, $\left.0.8 \mathrm{~mL} / \mathrm{min}, 20^{\circ} \mathrm{C}, 254 \mathrm{~nm}\right): t_{\mathrm{R}}($ minor $)=34.5 \mathrm{~min}, t_{\mathrm{R}}$ (major $)=39.8$ $\min$.

(S)-6-Methoxy-4',5'-dihydrospiro[indene-2,2'-pyran]-1,3',6'(3H)trione (3b)

Yield: $13 \mathrm{mg}$ (51\%); white solid; $\mathrm{mp} 109-111{ }^{\circ} \mathrm{C}$; $50 \%$ ee (determined by HPLC).

$[\alpha]_{D}^{20}+30\left(c 0.28, \mathrm{CHCl}_{3}\right)$.

IR (neat): 1743, 1734, 1701, 1275, 1028, 983, $748 \mathrm{~cm}^{-1}$.

${ }^{1} \mathrm{H}$ NMR $\left(500 \mathrm{MHz}, \mathrm{CDCl}_{3}\right): \delta=7.39(\mathrm{~d}, J=8.5 \mathrm{~Hz}, 1 \mathrm{H}), 7.29$ (dd, $J=8.5,2.5 \mathrm{~Hz}, 1 \mathrm{H}), 7.13(\mathrm{~d}, J=3.0 \mathrm{~Hz}, 1 \mathrm{H}), 3.83(\mathrm{~s}, 3 \mathrm{H}), 3.71-3.61(\mathrm{~m}$, $2 \mathrm{H}), 3.23$ (d, $J=16.5 \mathrm{~Hz}, 1 \mathrm{H}), 3.03-2.96(\mathrm{~m}, 1 \mathrm{H}), 2.85-2.81(\mathrm{~m}, 2 \mathrm{H})$.

${ }^{13} \mathrm{C}$ NMR $\left(125 \mathrm{MHz}, \mathrm{CDCl}_{3}\right): \delta=201.8,197.2,169.0,160.1,145.0$, 133.5, 126.9, 126.5, 106.5, 93.3, 55.7, 38.3, 33.4, 27.1.

HRMS (ESI): $\mathrm{m} / z$ calcd for $\mathrm{C}_{14} \mathrm{H}_{13} \mathrm{O}_{5}[\mathrm{M}+\mathrm{H}]^{+}$: 261.0757; found: 261.0761.

HPLC (YMC Chiral Amylose-C S-5 $\mu \mathrm{m}(25 \mathrm{~cm}$ ), hexane/ $i$-PrOH, 90:10, $\left.0.8 \mathrm{~mL} / \mathrm{min}, 10{ }^{\circ} \mathrm{C}, 254 \mathrm{~nm}\right): t_{\mathrm{R}}($ minor $)=65.4 \mathrm{~min}, t_{\mathrm{R}}($ major $)=70.4$ $\min$.

(S)-6-Methyl-4',5'-dihydrospiro[indene-2,2'-pyran]-1,3',6'(3H)-trione (3c)

Yield: $13 \mathrm{mg}$ (54\%); white solid; $\mathrm{mp} 166-168^{\circ} \mathrm{C} ; 61 \%$ ee (determined by HPLC).

$[\alpha]_{D}^{20}+18\left(\right.$ c $\left.0.21, \mathrm{CHCl}_{3}\right)$.

IR (neat): 2924, 1701, 1616, 1575, 1494, 1220, 1097, 831, $748 \mathrm{~cm}^{-1}$.

${ }^{1} \mathrm{H}$ NMR $\left(500 \mathrm{MHz}, \mathrm{CDCl}_{3}\right): \delta=7.54(\mathrm{~s}, 1 \mathrm{H}), 7.52(\mathrm{~d}, J=7.5 \mathrm{~Hz}, 1 \mathrm{H})$, $7.39(\mathrm{~d}, J=7.5 \mathrm{~Hz}, 1 \mathrm{H}), 3.74-3.64(\mathrm{~m}, 2 \mathrm{H}), 3.25(\mathrm{~d}, J=16.5 \mathrm{~Hz}, 1 \mathrm{H})$, 3.03-2.96 (m, $1 \mathrm{H}), 2.85-2.80(\mathrm{~m}, 2 \mathrm{H}), 2.41(\mathrm{~s}, 3 \mathrm{H})$.

${ }^{13} \mathrm{C}$ NMR $\left(125 \mathrm{MHz}, \mathrm{CDCl}_{3}\right): \delta=201.9,197.3,169.1,149.4,138.8$, $138.3,132.6,125.9,125.5,93.1,38.6,33.4,27.1,21.1$.

HRMS (ESI): $\mathrm{m} / z$ calcd for $\mathrm{C}_{14} \mathrm{H}_{13} \mathrm{O}_{4}[\mathrm{M}+\mathrm{H}]^{+}$: 245.0808; found: 245.0811.

HPLC (YMC Chiral Amylose-C S-5 $\mu \mathrm{m}(25 \mathrm{~cm})$, hexane/i-PrOH, 85:15, $\left.0.8 \mathrm{~mL} / \mathrm{min}, 20^{\circ} \mathrm{C}, 254 \mathrm{~nm}\right): t_{\mathrm{R}}($ minor $)=33.5 \mathrm{~min}, t_{\mathrm{R}}($ major $)=37.1$ $\min$. 
(S)-6-Chloro-4',5'-dihydrospiro[indene-2,2'-pyran]-1,3',6'(3H)-trione (3d)

Yield: $10 \mathrm{mg}$ (40\%); white solid; $\mathrm{mp} 172-174{ }^{\circ} \mathrm{C}$; $68 \%$ ee (determined by HPLC).

$[\alpha]_{\mathrm{D}}^{20}+14\left(\right.$ c $\left.0.24, \mathrm{CHCl}_{3}\right)$.

IR (neat): 2924, 2360, 1763, 1715, 1425, 1254, 1105, 982, $752 \mathrm{~cm}^{-1}$.

${ }^{1} \mathrm{H}$ NMR $\left(500 \mathrm{MHz}, \mathrm{CDCl}_{3}\right): \delta=7.71(\mathrm{~d}, J=2.0 \mathrm{~Hz}, 1 \mathrm{H}), 7.66(\mathrm{dd}$, $J=8.5,2.0 \mathrm{~Hz}, 1 \mathrm{H}), 7.46(\mathrm{~d}, J=8.0 \mathrm{~Hz}, 1 \mathrm{H}), 3.73(\mathrm{~d}, J=16.5 \mathrm{~Hz}, 1 \mathrm{H})$, 3.69-3.62 (m, $1 \mathrm{H}), 3.27$ (d, $J=17.0 \mathrm{~Hz}, 1 \mathrm{H}), 3.01(\mathrm{dt}, J=17.0,4.5 \mathrm{~Hz}$, $1 \mathrm{H}), 2.86-2.82(\mathrm{~m}, 2 \mathrm{H})$.

${ }^{13} \mathrm{C}$ NMR $\left(125 \mathrm{MHz}, \mathrm{CDCl}_{3}\right): \delta=201.4,196.2,168.6,145.0,137.0$, 135.1, 133.6, 127.4, 125.3, 92.8, 38.4, 33.3, 27.0.

HRMS (ESI): $m / z$ calcd for $\mathrm{C}_{13} \mathrm{H}_{10} \mathrm{ClO}_{4}[\mathrm{M}+\mathrm{H}]^{+}:$265.0262, 267.0233; found: $265.0266,267.0237$.

HPLC (YMC Chiral Amylose-C S-5 $\mu \mathrm{m}(25 \mathrm{~cm})$, hexane/i-PrOH, 90:10, $\left.1.0 \mathrm{~mL} / \mathrm{min}, 20^{\circ} \mathrm{C}, 254 \mathrm{~nm}\right): t_{\mathrm{R}}($ minor $)=28.8 \mathrm{~min}, t_{\mathrm{R}}($ major $)=38.1$ min.

\section{(S)-4',5'-Dihydrospiro[cyclopenta[a]naphthalene-2,2'-pyran]-} $1,3^{\prime}, 6^{\prime}(3 H)$-trione (3e)

Yield: $24 \mathrm{mg}$ (87\%); white solid; $\mathrm{mp} 200-202{ }^{\circ} \mathrm{C}$; 63\% ee (determined by HPLC).

$[\alpha]_{\mathrm{D}}{ }^{20}-26\left(\mathrm{c} 0.14, \mathrm{CHCl}_{3}\right)$.

IR (neat): 2920, 1699, 1573, 1517, 1417, 1312, 1180, 1155, 989, 826 $\mathrm{cm}^{-1}$.

${ }^{1} \mathrm{H}$ NMR $\left(500 \mathrm{MHz}, \mathrm{CDCl}_{3}\right): \delta=8.85(\mathrm{~d}, J=8.5 \mathrm{~Hz}, 1 \mathrm{H}), 8.16(\mathrm{~d}, J=8.5$ $\mathrm{Hz}, 1 \mathrm{H}), 7.92(\mathrm{~d}, J=8.5 \mathrm{~Hz}, 1 \mathrm{H}), 7.70(\mathrm{td}, J=8.0,1.0 \mathrm{~Hz}, 1 \mathrm{H}), 7.60(\mathrm{td}$, $J=8.0,1.0 \mathrm{~Hz}, 1 \mathrm{H}), 7.54(\mathrm{~d}, J=8.5 \mathrm{~Hz}, 1 \mathrm{H}), 3.85(\mathrm{~d}, J=17.0 \mathrm{~Hz}, 1 \mathrm{H})$, 3.84-3.76 (m, $1 \mathrm{H}), 3.42$ (d, J = 17.0 Hz, $1 \mathrm{H}), 3.07-3.01$ (m, $1 \mathrm{H}), 2.89-$ $2.85(\mathrm{~m}, 2 \mathrm{H})$.

${ }^{13} \mathrm{C}$ NMR $\left(125 \mathrm{MHz}, \mathrm{CDCl}_{3}\right): \delta=201.9,197.1,169.2,155.9,138.4$, 133.0, 129.9, 129.5, 128.6, 127.4, 126.9, 123.8, 123.0, 92.9, 39.1, 33.5, 27.2 .

HRMS (ESI): $m / z$ calcd for $\mathrm{C}_{17} \mathrm{H}_{13} \mathrm{O}_{4}[\mathrm{M}+\mathrm{H}]^{+}:$281.0808; found: 281.0812 .

HPLC (YMC Chiral Amylose-C S-5 $\mu \mathrm{m}(25 \mathrm{~cm})$, hexane/i-PrOH, 90:10, $\left.1.0 \mathrm{~mL} / \mathrm{min}, 20^{\circ} \mathrm{C}, 254 \mathrm{~nm}\right): t_{\mathrm{R}}($ minor $)=28.8 \mathrm{~min}, t_{\mathrm{R}}($ major $)=38.1$ min.

(S)-3,4,4',5'-Tetrahydro-1H-spiro[naphthalene-2,2'-pyran]-1,3',6'trione (3f)

Yield: $14 \mathrm{mg}$ (58\%); white solid; $\mathrm{mp} 96-98{ }^{\circ} \mathrm{C}$; $47 \%$ ee (determined by HPLC).

$[\alpha]_{\mathrm{D}}^{20}+11\left(\right.$ c $\left.0.17, \mathrm{CHCl}_{3}\right)$.

${ }^{1} \mathrm{H}$ NMR $\left(400 \mathrm{MHz}, \mathrm{CDCl}_{3}\right): \delta=7.98(\mathrm{dd}, J=7.6,1.2 \mathrm{~Hz}, 1 \mathrm{H}), 7.57(\mathrm{td}$, $J=7.6,1.2 \mathrm{~Hz}, 1 \mathrm{H}), 7.35$ (t, $J=7.6 \mathrm{~Hz}, 1 \mathrm{H}), 7.29(\mathrm{~d}, J=8.0 \mathrm{~Hz}, 1 \mathrm{H})$, 3.40-3.32 (m, $1 \mathrm{H}), 3.28-3.14(\mathrm{~m}, 2 \mathrm{H}), 3.00-2.83$ ( $\mathrm{m}, 2 \mathrm{H}), 2.75-2.63$ $(\mathrm{m}, 2 \mathrm{H}), 2.49-2.41(\mathrm{~m}, 1 \mathrm{H})$.

${ }^{13} \mathrm{C}$ NMR $\left(100 \mathrm{MHz}, \mathrm{CDCl}_{3}\right): \delta=203.5,191.1,169.3,144.1,135.1$, $129.4,128.9,128.7,127.2,88.2,34.1,31.6,27.7,24.7$.

HRMS (ESI): $m / z$ calcd for $\mathrm{C}_{14} \mathrm{H}_{13} \mathrm{O}_{4}[\mathrm{M}+\mathrm{H}]^{+}:$245.0808; found: 245.0811.

HPLC (YMC Chiral Amylose-C S-5 $\mu \mathrm{m}(25 \mathrm{~cm})$, hexane/i-PrOH, 90:10, $\left.0.8 \mathrm{~mL} / \mathrm{min}, 20^{\circ} \mathrm{C}, 254 \mathrm{~nm}\right): t_{\mathrm{R}}$ (major) $=34.2 \mathrm{~min}, t_{\mathrm{R}}($ minor $)=41.4$ $\min$.

\section{6-0xaspiro[4.5]decane-1,7,10-trione (3g)}

Yield: $7 \mathrm{mg}$ (36\%); yellow oil; 0\% ee (determined by HPLC).

IR (neat): 2924, 1743, 1713, 1456, 1265, 1142, 1103, 1051, 1009, 970, $872 \mathrm{~cm}^{-1}$.

${ }^{1} \mathrm{H}$ NMR (500 MHz, $\left.\mathrm{CDCl}_{3}\right): \delta=3.34-3.25(\mathrm{~m}, 1 \mathrm{H}), 2.94-2.87(\mathrm{~m}, 1 \mathrm{H})$, 2.80-2.68 (m, 2 H), 2.65-2.57 ( $\mathrm{m}, 1 \mathrm{H}), 2.55-2.40$ ( $\mathrm{m}, 2 \mathrm{H}), 2.30-2.07$ $(\mathrm{m}, 3 \mathrm{H})$

${ }^{13} \mathrm{C}$ NMR $\left(125 \mathrm{MHz}, \mathrm{CDCl}_{3}\right): \delta=209.9,203.2,168.9,91.7,35.8,34.3$, $33.8,27.0,18.2$

HRMS (ESI): $m / z$ calcd for $\mathrm{C}_{9} \mathrm{H}_{11} \mathrm{O}_{4}[\mathrm{M}+\mathrm{H}]^{+}$: 183.0652; found: 183.0651 .

HPLC (YMC Chiral Amylose-C S-5 $\mu \mathrm{m}(25 \mathrm{~cm}$ ), hexane/i-PrOH, 90:10, $\left.1.0 \mathrm{~mL} / \mathrm{min}, 20^{\circ} \mathrm{C}, 221 \mathrm{~nm}\right): t_{\mathrm{R}}(1)=14.2 \mathrm{~min}, t_{\mathrm{R}}(2)=21.3 \mathrm{~min}$

\section{(R)-Spiro[indene-2,3'-isochroman]-1,1',4'(3H)-trione (3h)}

Yield: $18 \mathrm{mg}$ (64\%); white solid; $\mathrm{mp} 140-142{ }^{\circ} \mathrm{C} ; 67 \%$ ee (determined by HPLC).

$[\alpha]_{D}^{20}-34\left(c 0.51, \mathrm{CHCl}_{3}\right)$.

IR (neat): 1738, 1690, 1595, 1421, 1263, 1213, 1101, 1006, 935, 851 $\mathrm{cm}^{-1}$

${ }^{1} \mathrm{H}$ NMR $\left(500 \mathrm{MHz}, \mathrm{CDCl}_{3}\right): \delta=8.38(\mathrm{dd}, J=8.0,1.0 \mathrm{~Hz}, 1 \mathrm{H}), 8.00(\mathrm{dd}$, $J=7.5,1.0 \mathrm{~Hz}, 1 \mathrm{H}), 7.91(\mathrm{td}, J=7.5,1.0 \mathrm{~Hz}, 1 \mathrm{H}), 7.80$ (td, $J=8.0,1.0$ $\mathrm{Hz}, 1 \mathrm{H}), 7.75-7.69(\mathrm{~m}, 2 \mathrm{H}), 7.59(\mathrm{dt}, J=8.0,1.0 \mathrm{~Hz}, 1 \mathrm{H}), 7.44(\mathrm{t}$, $J=8.0 \mathrm{~Hz}, 1 \mathrm{H}), 4.20(\mathrm{~d}, J=16.5 \mathrm{~Hz}, 1 \mathrm{H}), 3.47(\mathrm{~d}, J=16.5 \mathrm{~Hz}, 1 \mathrm{H})$.

${ }^{13} \mathrm{C}$ NMR $\left(125 \mathrm{MHz}, \mathrm{CDCl}_{3}\right): \delta=195.5,187.9,161.6,152.2,137.0$, $135.9,134.2,131.5,130.9,130.5,129.5,128.6,126.6,126.4,126.0$, 94.0, 37.4

HRMS (ESI): $m / z$ calcd for $\mathrm{C}_{17} \mathrm{H}_{11} \mathrm{O}_{4}[\mathrm{M}+\mathrm{H}]^{+}$: 279.0652; found: 279.0655 .

HPLC (YMC Chiral Amylose-C S-5 $\mu \mathrm{m}(25 \mathrm{~cm}$ ), hexane/i-PrOH, 90:10, $\left.0.8 \mathrm{~mL} / \mathrm{min}, 20^{\circ} \mathrm{C}, 254 \mathrm{~nm}\right): t_{R}($ major $)=32.2 \mathrm{~min}, t_{R}($ minor $)=35.3$ min.

(S)-Methyl 4-(2-Hydroxy-1-oxo-2,3-dihydro-1H-inden-2-yl)-4oxobutanoate (5a)

Yield: $17 \mathrm{mg}$ (66\%); colorless oil; 31\% ee (determined by HPLC).

$[\alpha]_{\mathrm{D}}^{20}+19\left(\mathrm{c} \mathrm{0.32}, \mathrm{CHCl}_{3}\right)$.

IR (neat): 1726, 1705, 1609, 1211, 1092, $733 \mathrm{~cm}^{-1}$.

${ }^{1} \mathrm{H}$ NMR $\left(500 \mathrm{MHz}, \mathrm{CDCl}_{3}\right): \delta=7.82(\mathrm{~d}, J=9.0 \mathrm{~Hz}, 1 \mathrm{H}), 7.70(\mathrm{t}, J=7.5$ $\mathrm{Hz}, 1 \mathrm{H}), 7.55(\mathrm{~d}, J=7.5 \mathrm{~Hz}, 1 \mathrm{H}), 7.46(\mathrm{t}, J=7.5 \mathrm{~Hz}, 1 \mathrm{H}), 4.42(\mathrm{~s}, 1 \mathrm{H})$, 3.82 (d, $J=17.5 \mathrm{~Hz}, 1 \mathrm{H}), 3.65(\mathrm{~s}, 3 \mathrm{H}), 3.24(\mathrm{~d}, J=17.5 \mathrm{~Hz}, 1 \mathrm{H}), 2.82-$ 2.66 (m, $2 \mathrm{H}), 2.54-2.43$ ( $\mathrm{m}, 2 \mathrm{H}$ ).

${ }^{13} \mathrm{C}$ NMR $\left(125 \mathrm{MHz}, \mathrm{CDCl}_{3}\right): \delta=205.1,201.5,172.7,152.2,136.5$, $134.3,128.5,126.8,125.3,87.1,52.0,38.9,31.3,27.5$.

HRMS (ESI): $m / z$ calcd for $\mathrm{C}_{14} \mathrm{H}_{15} \mathrm{O}_{5}[\mathrm{M}+\mathrm{H}]^{+}$: 263.0914; found: 263.0916.

HPLC (YMC Chiral Amylose-C S-5 $\mu \mathrm{m}(25 \mathrm{~cm}$ ), hexane/i-PrOH, 90:10, $\left.0.8 \mathrm{~mL} / \mathrm{min}, 20^{\circ} \mathrm{C}, 254 \mathrm{~nm}\right): t_{\mathrm{R}}($ minor $)=22.6 \mathrm{~min}, t_{\mathrm{R}}($ major $)=27.3$ $\min$.

(S)-Methyl 4-(2-Methoxy-1-oxo-2,3-dihydro-1H-inden-2-yl)-4oxobutanoate (5b)

Yield: $10 \mathrm{mg}$ (38\%); colorless oil; 77\% ee (determined by HPLC).

$[\alpha]_{\mathrm{D}}^{20}+26\left(c \mathrm{0} .45, \mathrm{CHCl}_{3}\right)$.

IR (neat): 2953, 2926, 1730, 1707, 1609, 1458, 1209, 1132, 1101, $1020,771 \mathrm{~cm}^{-1}$. 
${ }^{1} \mathrm{H}$ NMR $\left(500 \mathrm{MHz}, \mathrm{CDCl}_{3}\right): \delta=7.73(\mathrm{~d}, J=7.5 \mathrm{~Hz}, 1 \mathrm{H}), 7.64(\mathrm{t}, J=7.5$ $\mathrm{Hz}, 1 \mathrm{H}), 7.50(\mathrm{~d}, J=8.0 \mathrm{~Hz}, 1 \mathrm{H}), 7.39(\mathrm{t}, J=7.5 \mathrm{~Hz}, 1 \mathrm{H}), 3.68(\mathrm{~d}$, $J=17.0 \mathrm{~Hz}, 1 \mathrm{H}), 3.64(\mathrm{~s}, 3 \mathrm{H}), 3.41(\mathrm{~s}, 3 \mathrm{H}), 3.23-3.08$ (m, $3 \mathrm{H}), 2.62-$ $2.46(\mathrm{~m}, 2 \mathrm{H})$.

${ }^{13} \mathrm{C}$ NMR $\left(125 \mathrm{MHz}, \mathrm{CDCl}_{3}\right): \delta=205.8,199.5,173.0,152.5,136.2$, $134.1,128.0,126.5,124.8,93.7,53.6,51.7,33.6,32.6,27.5$.

HRMS (ESI): $m / z$ calcd for $\mathrm{C}_{15} \mathrm{H}_{17} \mathrm{O}_{5}[\mathrm{M}+\mathrm{H}]^{+}$: 277.1071; found: 277.1075 .

HPLC (Daicel Chiralcel OD-H column $(25 \mathrm{~cm})$, hexane/i-PrOH, 95:5, $\left.1.0 \mathrm{~mL} / \mathrm{min}, 20^{\circ} \mathrm{C}, 254 \mathrm{~nm}\right): t_{\mathrm{R}}($ minor $)=20.3 \mathrm{~min}, t_{\mathrm{R}}($ major $)=24.1$ min.

\section{(S)-Methyl 4-(2-Ethoxy-1-oxo-2,3-dihydro-1H-inden-2-yl)-4-} oxobutanoate (5c)

Yield: $12 \mathrm{mg}$ (40\%); colorless oil; 79\% ee (determined by HPLC).

$[\alpha]_{\mathrm{D}}^{20}+31\left(\mathrm{c} \mathrm{0.70}, \mathrm{CHCl}_{3}\right)$.

IR (neat): 2924, 1728, 1707, 1609, 1437, 1213, 1161, 1105, 1038, 772 $\mathrm{cm}^{-1}$.

${ }^{1} \mathrm{H}$ NMR $\left(400 \mathrm{MHz}, \mathrm{CDCl}_{3}\right): \delta=7.72(\mathrm{~d}, J=7.6 \mathrm{~Hz}, 1 \mathrm{H}), 7.63(\mathrm{t}, J=8.0$ $\mathrm{Hz}, 1 \mathrm{H}), 7.48(\mathrm{~d}, J=7.6 \mathrm{~Hz}, 1 \mathrm{H}), 7.38(\mathrm{t}, J=7.6 \mathrm{~Hz}, 1 \mathrm{H}), 3.70(\mathrm{~d}$, $J=17.2 \mathrm{~Hz}, 1 \mathrm{H}), 3.64(\mathrm{~s}, 3 \mathrm{H}), 3.60-3.45(\mathrm{~m}, 2 \mathrm{H}), 3.24-3.10(\mathrm{~m}, 3 \mathrm{H})$, $2.62-2.45(\mathrm{~m}, 2 \mathrm{H}), 1.30(\mathrm{t}, J=6.8 \mathrm{~Hz}, 3 \mathrm{H})$.

${ }^{13} \mathrm{C}$ NMR $\left(100 \mathrm{MHz}, \mathrm{CDCl}_{3}\right): \delta=206.1,199.7,173.1,152.6,136.1$, 134.0, 128.0, 126.4, 124.8, 93.4, 61.7, 51.7, 34.3, 32.6, 27.5, 15.6.

HRMS (ESI): $m / z$ calcd for $\mathrm{C}_{16} \mathrm{H}_{18} \mathrm{O}_{5} \mathrm{Na}[\mathrm{M}+\mathrm{Na}]^{+}$: 313.1052; found: 313.1061.

HPLC (Daicel Chiralcel OB-H column $(25 \mathrm{~cm})$, hexane/i-PrOH, 90:10, $1.0 \mathrm{~mL} / \mathrm{min}, 20^{\circ} \mathrm{C}, 254 \mathrm{~nm}$ ): $t_{\mathrm{R}}$ (major) $=22.2 \mathrm{~min}, t_{\mathrm{R}}($ minor $)=36.8$ $\min$.

\section{Funding Information}

This work was supported by the China Scholarship Council (No. 201608140185). Support from Cardiff University and Taiyuan University of Technology is gratefully acknowledged.

\section{Acknowledgment}

We thank the EPSRC National Mass Spectrometry Facility, Swansea, for mass spectrometric analysis.

\section{Supporting Information}

Supporting information for this article is available online at https://doi.org/10.1055/s-0037-1610373.

\section{References}

(1) Current address: College of Chemistry and Chemical Engineering, Taiyuan University of Technology, Yingze West Street 79, Taiyuan 030024, P. R. of China.

(2) For reviews and books, see: (a) Flores, A.; Cots, E.; Bergès, J.; Muñiz, K. Adv. Synth. Catal. 2018, in press; DOI: 10.1002/adsc.201800521. (b) Yoshimura, A.; Zhdankin, V. V. Chem. Rev. 2016, 116, 3328. (c) Wirth, T. Hypervalent Iodine
Chemistry; Berlin: Springer-Verlag, 2016. For recent publications on chiral iodine(III) reagents, see: (d) Companys, S.; Peixoto, P. A.; Bosset, C.; Chassaing, S.; Miqueu, K.; Sotiropoulos, J. M.; Pouységu, L.; Quideau, S. Chem. Eur. J. 2017, 23, 13309. (e) Shimogaki, M.; Fujita, M.; Sugimura, T. Angew. Chem. Int. Ed. 2016, 55, 15797. (f) Kong, W.; Feige, P.; Haro, T.; Nevado, C. Angew. Chem. Int. Ed. 2013, 52, 2469.

(3) (a) Fujita, M. Tetrahedron Lett. 2017, 58, 4409. (b) Woerly, E. M.; Banik, S. M.; Jacobsen, E. N. J. Am. Chem. Soc. 2016, 138, 13858. (c) Muñiz, K.; Barreiro, L.; Romero, R. M.; Martínez, C. J. Am. Chem. Soc. 2017, 139, 4354. (d) Suzuki, S.; Kamo, T.; Fukushi, K.; Hiramatsu, T.; Tokunaga, E.; Dohi, T.; Kita, Y.; Shibata, N. Chem. Sci. 2014, 5, 2754. (e) Haubenreisser, S.; Wöste, T. H.; Martínez, C.; Ishihara, K.; Muñiz, K. Angew. Chem. Int. Ed. 2016, 55, 413. (f) Gelis, C.; Dumoulin, A.; Bekkaye, M.; Neuville, L.; Masson, G. Org. Lett. 2017, 19, 278.

(4) (a) Dohi, T.; Maruyama, A.; Takenaga, N.; Senami, K.; Minamitsuji, Y.; Fujioka, H.; Caemmerer, S. B.; Kita, Y. Angew. Chem. Int. Ed. 2008, 47, 3787. (b) Uyanik, M.; Yasui, T.; Ishihara, K. Angew. Chem. Int. Ed. 2010, 49, 2175. (c) Uyanik, M.; Yasui, T.; Ishihara, K. Angew. Chem. Int. Ed. 2013, 52, 9215. (d) Uyanik, M.; Sasakura, N.; Mizuno, M.; Ishihara, K. ACS Catal. 2017, 7, 872. (e) Dohi, T.; Sasa, H.; Miyazaki, K.; Fujitake, M.; Takenaga, N.; Kita, Y. J. Org. Chem. 2017, 82, 11954. (f) Uyanik, M.; Yasui, T.; Ishihara, K. J. Org. Chem. 2017, 82, 11946.

(5) Banik, S. M.; Medley, J. W.; Jacobsen, E. N. Science 2016, 353, 51.

(6) (a) Wu, H.; He, Y.-P.; Xu, L.; Zhang, D.-Y.; Gong, L.-Z. Angew. Chem. Int. Ed. 2014, 53, 3466. (b) Zhang, D.-Y.; Xu, L.; Wu, H.; Gong, L.-Z. Chem. Eur. J. 2015, 21, 10314. (c) Cao, Y.; Zhang, X.; Lin, G.; Negrerie, D. Z.; Du, Y. Org. Lett. 2016, 18, 5580. (d) Hu, B.; Cao, Y.; Zhang, B.; Negrerie, D. Z.; Du, Y. Adv. Synth. Catal. 2017, 359, 2542.

(7) (a) Farooq, U.; Schäfer, S.; Shah, A.-H. A.; Freudendahl, D. M.; Wirth, T. Synthesis 2010, 1023. (b) Rodríguez, A.; Moran, W. J. Synthesis 2012, 44, 1178.

(8) For reviews on electrochemistry, see: (a) Möhle, S.; Zirbes, M.; Rodrigo, E.; Gieshoff, T.; Wiebe, A.; Waldvogel, S. R. Angew. Chem. Int. Ed. 2018, 57, 6018. (b) Yan, M.; Kawamata, Y.; Baran, P. S. Chem. Rev. 2017, 117, 13230. (c) Francke, R.; Little, R. D. Chem. Soc. Rev. 2014, 43, 2492. (d) Yoshida, J.; Kataoka, K.; Horcajada, R.; Nagaki, A. Chem. Rev. 2008, 108, 2265. (e) Sperry, J. B.; Wright, D. L. Chem. Soc. Rev. 2006, 35, 605. (f) Moeller, K. D. Tetrahedron 2000, 56, 9527. For selected recent publications on electroorganic chemistry, see: (g) Kawamata, Y.; Yan, M.; Liu, Z.; Bao, D.-H.; Chen, J.; Starr, J. T.; Baran, P. S. J. Am. Chem. Soc. 2017, 139, 7448. (h) Horn, E. J.; Rosen, B. R.; Chen, Y.; Tang, J.; Chen, K.; Eastgate, M. D.; Baran, P. S. Nature 2016, 533, 77. (i) Wiebe, A.; Lips, S.; Schollmeyer, D.; Franke, R.; Waldvogel, S. R. Angew. Chem. Int. Ed. 2017, 56, 14727. (j) Schulz, L.; Enders, M.; Elsler, B.; Schollmeyer, D.; Dyballa, K. M.; Franke, R.; Waldvogel, S. R. Angew. Chem. Int. Ed. 2017, 56, 4877. (k) Wu, Z.-J.; Xu, H.-C. Angew. Chem. Int. Ed. 2017, 56, 4734. (l) Zhao, H.-B.; Liu, Z.-J.; Song, J.; Xu, H.-C. Angew. Chem. Int. Ed. 2017, 56, 12732. (m) Badalyan, A.; Stahl, S. S. Nature 2016, 535, 406. (n) Yang, Q.L.; Li, Y.-Q.; Ma, C.; Fang, P.; Zhang, X.-J.; Mei, T.-S. J. Am. Chem. Soc. 2017, 139, 3293. (o) Sauermann, N.; Meyer, T. H.; Tian, C.; Ackermann, L. J. Am. Chem. Soc. 2017, 139, 18452. (p) Francke, R.; Little, R. D. J. Am. Chem. Soc. 2014, 136, 427.

(9) Zhang, S.; Lian, F.; Xue, M.; Qin, T.; Li, L.; Zhang, X.; Xu, K. Org. Lett. 2017, 19, 6622. 
(10) For molecules containing chiral keto lactones, see: (a) Nakayama, M.; Fukuoka, Y.; Nozaki, H.; Matsuo, A.; Hayashi, S. Chem. Lett. 1980, 1243. (b) Aramaki, Y.; Chiba, K.; Tada, M. Phytochemistry 1995, 38, 1419. (c) Yan, B.-F.; Fang, S.-T.; Li, W.Z.; Liu, S.-J.; Wang, J.-H.; Xia, C.-H. Nat. Prod. Res. 2015, 29, 2013.

(11) Schmidt, H.; Meinert, H. Angew. Chem. 1960, 72, 109.

(12) (a) Fuchigami, T.; Fujita, T. J. Org. Chem. 1994, 59, 7190. (b) Sawamura, T.; Kuribayashi, S.; Inagi, S.; Fuchigami, T. Org. Lett. 2010, 12, 644. (c) Sawamura, T.; Kuribayashi, S.; Inagi, S.; Fuchigami, T. Adv. Synth. Catal. 2010, 352, 2757.

(13) (a) Inoue, K.; Ishikawa, Y.; Nishiyama, S. Org. Lett. 2010, 12, 436. (b) Kajiyama, D.; Inoue, K.; Ishikawa, Y.; Nishiyama, S. Tetrahedron 2010, 66, 9779. (c) Broese, T.; Francke, R. Org. Lett. 2016, 18, 5896. (d) Koleda, O.; Broese, T.; Noetzel, J.; Roemelt, M.; Suna, E.; Francke, R. J. Org. Chem. 2017, 82, 11669.

(14) (a) Francke, R.; Broese, T.; Rösel, A. In Patai's Chemistry of Functional Groups, Hypervalent Halogen Compounds; Marek, I.; Olofsson, B.; Rappoport, Z., Ed.; John Wiley \& Sons, Ltd: Chichester, 2018. (b) Elsherbini, M.; Wirth, T. Chem. Eur. J. 2018, 24, 13399.

(15) (a) Qurban, J.; Elsherbini, M.; Wirth, T. J. Org. Chem. 2017, 82, 11872. (b) Malmedy, F.; Wirth, T. Chem. Eur. J. 2016, 22, 16072. (c) Mizar, P.; Niebuhr, R.; Hutchings, M.; Farooq, U.; Wirth, T. Chem. Eur. J. 2016, 22, 1614. (d) Brown, M.; Kumar, R.; Rehbein, J.; Wirth, T. Chem. Eur. J. 2016, 22, 4030. (e) Mizar, P.; Wirth, T. Angew. Chem. Int. Ed. 2014, 53, 5993. (f) Farid, U.; Malmedy, F.; Claveau, R.; Albers, L.; Wirth, T. Angew. Chem. Int. Ed. 2013, 52, 7018. (g) Farid, U.; Wirth, T. Angew. Chem. Int. Ed. 2012, 51, 3462.

(16) For selected enantioselective electrochemical processes, see: (a) Fu, N.; Li, L.; Yang, Q.; Luo, S. Org. Lett. 2017, 19, 2122. (b) Jensen, K. L.; Franke, P. T.; Nielsen, L. T.; Daasbjerg, K.; Jørgensen, K. A. Angew. Chem. Int. Ed. 2010, 49, 129. (c) Nguyen, B. H.; Redden, A.; Moeller, K. D. Green Chem. 2014, 16, 69.
(17) (a) Folgueiras-Amador, A. A.; Qian, X.-Y.; Xu, H.-C.; Wirth, T. Chem. Eur. J. 2018, 24, 487. (b) Folgueiras-Amador, A. A.; Philipps, K.; Guilbaud, S.; Poelakker, J.; Wirth, T. Angew. Chem. Int. Ed. 2017, 56, 15446. (c) Arai, K.; Wirth, T. Org. Process Res. Dev. 2014, 18, 1377.

(18) CCDC 1834441 (3a) contains the supplementary crystallographic data for this paper. The data can be obtained free of charge from The Cambridge Crystallographic Data Centre via www.ccdc.cam.ac.uk/getstructures.

(19) For recent electrochemical oxidations of amides and benzyl ethers, see: (a) Xu, F.; Qian, X.-Y.; Li, Y.-J.; Xu, H.-C. Org. Lett. 2017, 19, 6332. (b) Gieshoff, T.; Kehl, A.; Schollmeyer, D.; Moeller, K. D.; Waldvogel, S. R. J. Am. Chem. Soc. 2017, 139, 12317. (c) Xiong, P.; Xu, H.-H.; Xu, H.-C. J. Am. Chem. Soc. 2017, 139, 2956. (d) Rafiee, M.; Wang, F.; Hruszkewycz, D. P.; Stahl, S. S. J. Am. Chem. Soc. 2018, 140, 22.

(20) For reviews on flow electrolysis, see: (a) Watts, K.; Baker, A.; Wirth, T. J. Flow Chem. 2014, 4, 2. (b) Folgueiras-Amador, A. A.; Wirth, T. In Science of Synthesis: Flow Chemistry in Organic Synthesis; Jamison, T. F.; Koch, G., Ed.; Georg Thieme Verlag KG: Stuttgart, 2018. (c) Pletcher, D.; Green, R. A.; Brown, R. C. D. Chem. Rev. 2018, 118, 4573. (d) Atobe, M.; Tateno, H.; Matsumura, Y. Chem. Rev. 2018, 118, 4541. For selected publications, see: (e) Gütz, C.; Stenglein, A.; Waldvogel, S. R. Org. Process Res. Dev. 2017, 21, 771. (f) Green, R. A.; Brown, R. C. D.; Pletcher, D.; Harji, B. Org. Process Res. Dev. 2015, 19, 1424. (g) Kabeshov, M. A.; Musio, B.; Murray, P. R. D.; Browne, D. L.; Ley, S. V. Org. Lett. 2014, 16, 4618. (h) Green, R. A.; Pletcher, D.; Leach, S. G.; Brown, R. C. D. Org. Lett. 2016, 18, 1198. (i) Arai, T.; Tateno, H.; Nakabayashi, K.; Kashiwagi, T.; Atobe, M. Chem. Commun. 2015, 51, 4891. 